\title{
Modelling of fluid structure interactions in stenosed arteries: effect of plaque deformability
}

\author{
Tristan Belzacq†, Stéphane Avril*† Emmanuel Leriche ${ }^{+}$and Alexandre Delache ${ }^{+}$ \\ † Ecole Nationale Supérieure des Mines \\ Centre Ingénierie et Santé, \\ CNRS UMR 5146 - INSERM IFR 143 \\ 158 cours Fauriel \\ 42023 SAINT-ETIENNE cedex 2 \\ FRANCE \\ ‡ Université Jean Monnet \\ Faculté des Sciences et Techniques \\ 23 rue du Docteur Paul Michelon \\ 42023 SAINT-ETIENNE cedex 2 \\ FRANCE
}

\begin{abstract}
*Corresponding author. Email: avril@emse.fr
Keywords: vascular, fluid structure interaction; stenosis, blood flow
\end{abstract}

\section{Introduction}

This paper deals with the study of fluid structure interactions in stenosed arteries. Stenoses in arteries are usually induced by the formation of an atheromatous plaque. This is a major issue of public health. Diagnoses based on simple image analysis (measurement of stenosis severity) are currently insufficient for reducing the impact of the cardio-vascular diseases, which may provoke a decrease of life expectancy for the 25-45 generation in the near future. Improved diagnoses should be devised for avoiding this issue. Therefore, there is a real medical and economical interest in developing tools and systems for determining and understanding the mechanical response of plaques in the arteries [1].

This mechanical response is mainly induced by the combination of shear and pressure loads applied by the blood at each heart beat. Several authors developed numerical models based on fluid structure interactions (FSI) for taking into account these effects [2,3]. Their main conclusion was that the vulnerability of atheromatous plaques may be explained by a concentration of stresses in the fibre cap that contains the lipid core of the plaque.

However, there is a large variability of plaque types among the patients and the models cited previously considered only very idealized geometry. No parametric study has ever been published about the effect of plaque type, plaque composition and plaque geometry on the mechanical response of the stenosed artery. The main objective of this paper is to address this issue.

\section{Methods}

A 2D axisymmetric model of a stenosed artery has been developed (Figure 1). It is composed of the healthy arterial wall outside (material 1), a lipid core (material 2), a fibre cap (material 3) containing the lipid core and a calcified inclusion (material 4). Each of this material is modelled as an elastic solid with a Poisson's ratio of 0.45 and a different Young's modulus $(\mathrm{E} 1=500 \mathrm{kPa}$, $\mathrm{E} 2=20 \mathrm{kPa}, \mathrm{E} 3=600 \mathrm{kPa}, \mathrm{E} 4=10 \mathrm{MPa})$.

Different plaque parameters can be tuned (Figure 1) for covering different types of atheromatous plaques reported in [4]: the elastic moduli of the constituents, the thickness of the fibre (e), the severity (S) of the stenosis (ratio between the radius of the healthy artery and the stenosed artery), the radius (Rem) of remodelling (externally bulging part of the artery), the length of the stenosis (L), location of the calcified inclusion.

Blood is modelled as an incompressible Newtonian fluid. FSI computations are run using the finite element (FE) method in Comsol software. Mesh updating of the fluid problem is based on an Arbitrary Eulerial Lagrangian (ALE) 
scheme. Boundary conditions for the fluid are a physiological Womersley velocity profile at the inlet, and a physiological pressure prescribed at the outlet.

\section{Results and conclusions}

The advantage of using a 2D axisymmetric model is that a large number of FE models could be run in a reasonable time. The analysis focuses on the following aspects of the computed responses:

- A1: maximum cyclic Von Mises equivalent strain in the fibre cap [5]

- A2: maximum cyclic shear stress

- A3: maximum radial and axial motion of the plaque during a cardiac cycle

- A4: length of the recirculation zone.

Results show that A1, A2, A3 and A4 increase when increasing $S$ and decreasing $L$. Indeed, for very slender plaques, a pinching occurs, resulting in increased deformations. This phenomenon is shown in Figure 2 on a severe short plaque where the deformed shape of the fibrous cap was plotted at the systolic time. The pinching phenomenon can also induce buckling in the fibrous cap for larger stenoses when e is smaller, as shown in Figure 3.

Increase of E3 and e is shown to reduce A1 and A3. The presence of a calcified inclusion is shown to increase A1 and A3, and hence to amplify the pinching phenomenon.

The interpretation of these results is under progress and it still has to be validated by an experimental study. However, the trends are that the effects of pressure and shear loads applied by the blood is amplified if plaques are very deformable. In other words, the most stable plaques are the least deformable ones. These trends are usually confirmed by the clinical practice.

Future work will address the definition a criterion of plaque vulnerability, based on plaque deformability. 3D models of the stenosed carotid bifurcation are also under development.

\section{References}

[1] Li, Z.-Y., Howarth, S., Trivedi, R., U-King-Im, J., Graves, M., Brown, A., Wang, L. and Gillard J. (2006) Stress analysis of carotid plaque rupture based on in vivo high resolution MRI. Journal of Biomechanics, 39, 2611-2622.

[2] Li. Z.-H. and Gillard J. (2007) Simulation of the interaction between blood flow and atherosclerotic plaque. In Conference of the IEEE EMBS.
[3] Tang, T., Teng, Z., Canton, G., Hatsukami, T., Dong, L., Huang, X., Yuan, L. Local critical stress correlates better than maximum stress with plaque morphological features linked to atherosclerotic plaque vulnerability: an in-vivo multi-patient study. BioMedical Engineering Online, 8:8-15.

[4] Cai, J.-M., Hatsukami, S., Ferguson, M.S., Small, R., Polissar, N.L., Yuan, C. (2002) Classification of human carotid atherosclerotic lesions with in vivo multicontrast magnetic resonance imaging. Journal of the American Heart Association, 1006:1368-1373.

[5] Ohayon, J., Teppaz, P., Finet, G., and Rioufol, G. (2001) In-vivo prediction of human coronary plaque rupture location using intravascular ultrasound and the finite element method, Coron Artery Dis, 12:655-63.

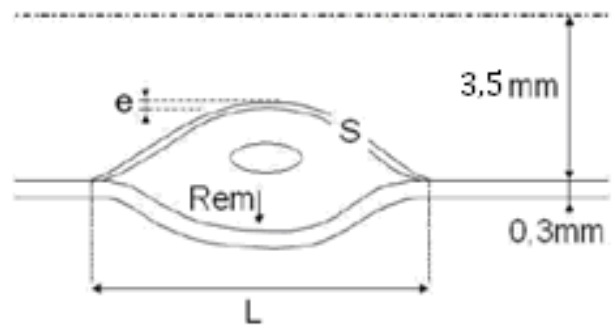

Figure 1. Geometrical model of the plaque: $\mathrm{e}=$ thickness of the fibre cap, $\mathrm{S}=$ severity of the stenosis, Rem=increase in external radius, $\mathrm{L}=$ length of the stenosis.

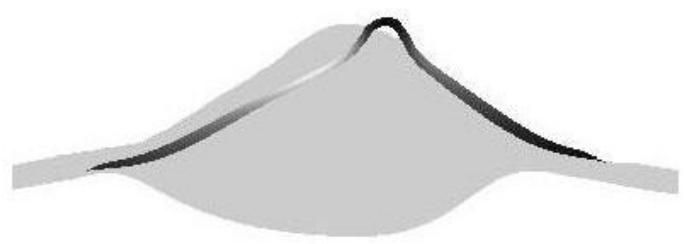

Figure 2. The deformed shape of the fibrous cap at systole (colorbar figures the stress level, from minimum=black to maximum=white) and the plaque deform shape at diastole in uniform grey.

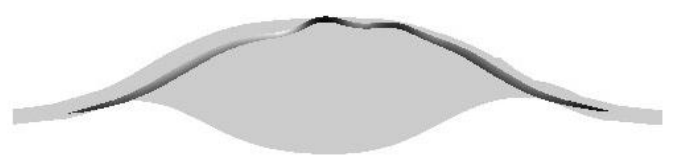

Figure 3. The deformed shape of the fibrous cap with buckling at systole (colorbar figures the stress level, from minimum=black to maximum=white) and the plaque deform shape at diastole in uniform grey. 